\title{
A Simple Strategy for Fall Events Detection
}

\author{
Fouzi Harrou $^{a}$, Member, IEEE, Nabil Zerrouki ${ }^{b}$ \\ ${ }^{a}$ King Abdullah University of Science and Technology, \\ CEMSE Division, Thuwal, 23955-6900, Saudi Arabia \\ Email: fouzi.harrou@kaust.edu.sa
}

\author{
Ying $\operatorname{Sun}^{a}$, Amrane Houacine ${ }^{b}$ \\ ${ }^{b}$ University of Sciences and Technology Houari Boumédienne \\ Algeria, LCPTS, Faculty of Electronics and Computer Science
}

\begin{abstract}
The paper concerns the detection of fall events based on human silhouette shape variations. The detection of fall events is addressed from the statistical point of view as an anomaly detection problem. Specifically, the paper investigates the multivariate exponentially weighted moving average (MEWMA) control chart to detect fall events. Towards this end, a set of ratios for five partial occupancy areas of the human body for each frame are collected and used as the input data to MEWMA chart. The MEWMA fall detection scheme has been successfully applied to two publicly available fall detection databases, the UR fall detection dataset (URFD) and the fall detection dataset (FDD). The monitoring strategy developed was able to provide early alert mechanisms in the event of fall situations.
\end{abstract}

\section{INTRODUCTION}

For seniors, falls present a serious risk of injury and an obstacle to independent living [1]. At the minimum one-third of seniors aged 65 years and over fall at least one time a year [2]. Consequences include fractures, injuries which can cause a loss of autonomy, and even death. Furthermore, a survey carried out by [3] showed that more that 1.6 million U.S. people are hospitalized each year due to fall injuries. Therefore, providing strategies to detect and prevent falls could improve quality of life. For this, we need a reliable effective fall detection system to monitor people particularly those at higher risk, such as, with special needs and the elderly [4]. By detecting falls early, greater safety can be achieved by quicker responses to potential emergencies.

Along past four decades, there has been growing interest in human fall detection for human health and safety [5], [6], [7], [8], [9], [10]. This demand was attracted the attention of researchers and engineers to develop several fall detection techniques [4]. Fall detection techniques could be split into two main families: non-computer vision based approaches and computer vision based approaches [4], [5], [11]. Non-computer-vision-based fall detection methods are usually rested on information captured by sensors such as acceleration and vibration sensors. This technique uses sound, vibrations, and human body movement to detect a fall [8], [9], [10], [12], [13]. However, many people find acceleration sensors inconvenient to wear and the sensors are easily affected by noise background limiting their accuracy by increasing number of false alarms. To surmount these limitations, computer vision-based fall detection methods are gaining in popularity because they rely on information obtained from images and videos [14], [15], [16], [17], [18].
Statistical process control is a major tool for checking the process quality by identifying abnormalities and to make sure that it works in healthy condition [19], [20], [21], [22]. Multivariate statistical monitoring charts were designed to inspect different variables simultaneously [23], [24], [25], [23]. This paper addresses the problem of detecting fall events within a statistical framework. The detection of fall events is addressed from the statistical point of view as an anomaly detection problem. This anomaly occurred at the moment of the occurrence of fall event. The multivariate exponentially weighted moving average (MEWMA) monitoring chart was chosen in this paper for fall detection because of its sensitivity to small changes in the parameters of a multivariate process. Further, the MEWMA is based on the weighted moving average of all available observations, a design that provides improved sensitivity to small changes in the mean of a multivariate process [19]. The purpose of this paper is to detect early enough the occurrence of a fall event in order to provide early alert mechanisms in the event of fall situations. The use of the MEWMA control chart may offer a more sensitive approach to detect fall events. The monitoring strategy developed was able to provide early alert mechanisms in the event of fall situations.

The following Section presents the image processing steps, including segmentation and features extraction. Section III briefly reviews how MEWMA anomaly detection is performed. Next, in Section IV, the performance of the proposed stategy is evaluated. Finally, Section V concludes this study.

\section{VISION-BASED FALL DETECTION APPROACHES}

The fall-detection algorithm based on visual monitoring includes four major steps: data acquisition or collection, image segmentation, feature extraction and fall detection (see Figure 1). The data acquisition step is in charge of recording specific variables to determine whether the person has suffered a fall. The role of the segmentation step is extracting body silhouette from the image sequence. And the feature extraction step determines discriminative information that are used as input in fall detection and classification steps. Then, fall detection phase decide about the presence and absence of fall event. 


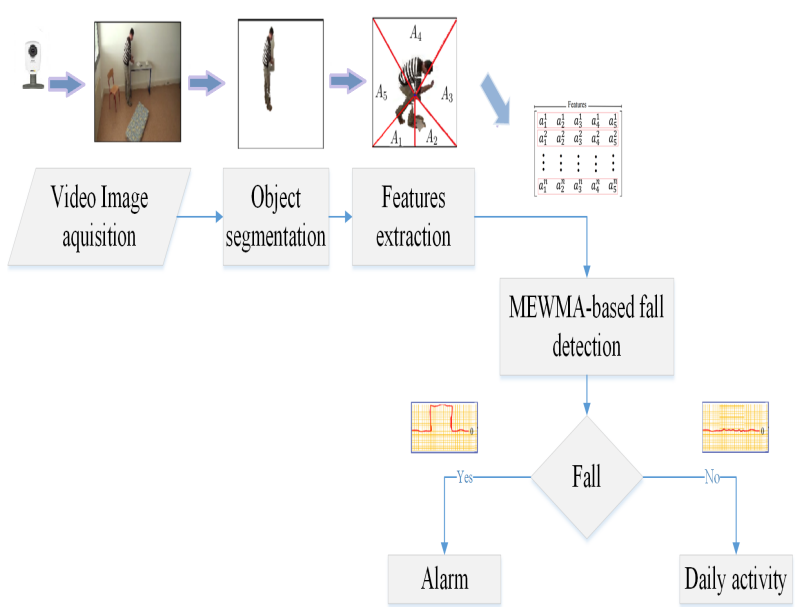

Fig. 1. Features used in fall detection procedure.

\section{A. Segmentation and preprocessing}

The segmentation phase consists of extracting body's silhouette from the input image sequence. In this work, human body is typically discriminated using background subtraction technique [26], [27]. In this case the aim is to subtract the image of interest from a reference background model [27]. The choice of this segmentation method was motivated by its efficiency even in presence of multi-component models [27]. Figure 2 illustrates an example of the background subtraction algorithm, where background and input frames are represented by the two images on the left, respectively, and the two images on the right, illustrate the respective results from background subtraction before and after applying morphological preprocessing.
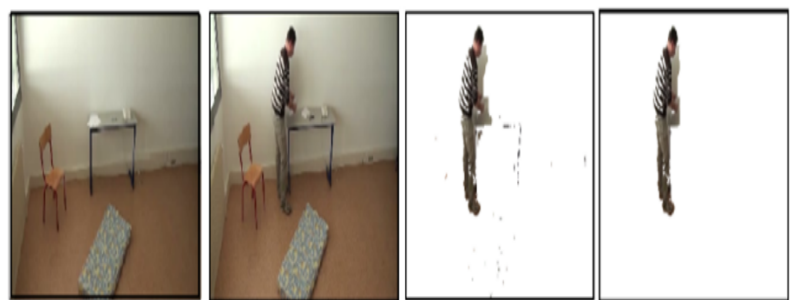

Fig. 2. Results of the background subtraction algorithm.

\section{B. Feature extraction:}

Feature extraction is the process by which discriminative human body attributes are extracted from the segmented frames. [28]. Many works have used the body's center of gravity as feature to detect a human fall fall by identifying that the distance between two centers of two successive images is significant [29] or that the body's gravity center is too close to the floor [13]. However, these approaches present some limitations especially when the position of the monitored person changes and when the floor is assimilated as background making it difficult to differentiate it from the monitored person in each frame [13].
These shortcomings motivated this paper, where the features extracted by computing five partial occupancy regions of the human body, which are determined by a partitioning based on the human gravity center (see Figure 3). This partitioning is performed by tracing five segments from the body's center of gravity. The first segment is vertical one, the second and third segments are located at $60^{\circ}$ on either side of the vertical segment, and the fourth and the fifth segments are situated at $120^{\circ}$ on either side of the third and fourth segments respectively (see Figure 3). These areas typically correspond to the action of body parts in a standing position (head, arms, and legs). A normalization phase is then required, where each area value is divided by the global body area. Given the total number of pixels making up the body area, $A$, and the number of pixels making up the partial areas $A_{i}, i=1 \ldots 5$, the normalized partial areas are given as:

$$
R_{i}=\frac{A_{i}}{A} .
$$

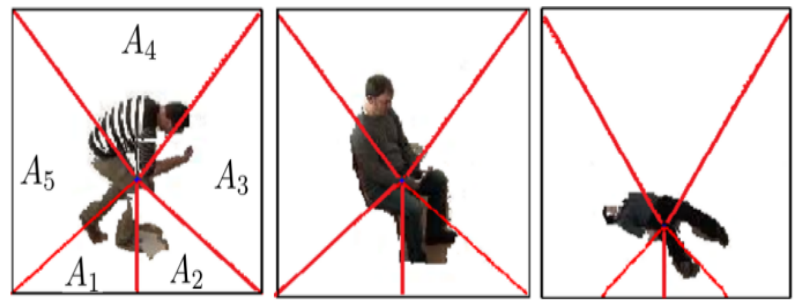

Fig. 3. Samples of human body areas partitioning.

Together these ratios constitute the feature vectors for use as indicators or descriptors in the fall detection process (see Figure 4). The extracted features are discriminative enough to describe human postures and not too computationally complex to allow a fast processing. Since frames of a video sequence are assimilated to an observation sequence, a set of ratios, which was computed for each frame, is then concatenated to form the entire feature vector corresponding to the video sequence. This vector is then exploited in fall detection phase as the input for MEWMA monitoring chart.

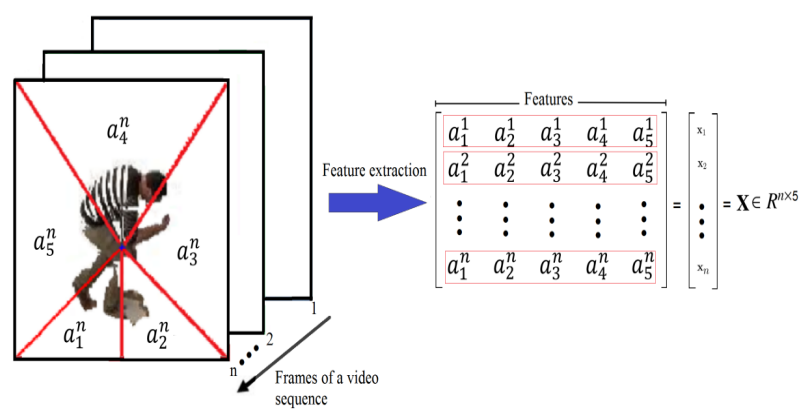

Fig. 4. Features used in fall detection procedure.

\section{Multivariate EWMA (MEWMA)-BASEd FAlL DETECTION SCHEME}

Fall detection can be formulated as an anomaly detection problem since the purpose in fall detection is making a binary 
decision about the occurrence of the fall (which corresponds to deciding between the presence and absence of anomalies in the extracted features). This anomaly occurs at the moment of the fall. When a person falls, a fall detection system would declare it as abnormal action. The main goal of this work is to detect the transition from the naturalistic situation to the fall situation early. Towards this end, the MEWMA monitoring chart will be used. The MEWMA scheme is best suited to detect small anomalies in the process mean [19], [30].

The MEWMA monitoring chart was initially proposed by Lowry et al. [31]. This chart is constructed based on exponential weighting of available observations. Assume that there is $\mathbf{X}_{t}=\left(X_{1}, X_{2}, \ldots, X_{m}\right)^{T}$, an $m$-dimensional set of observations at time $t$. The MEWMA charting statistic defined in as follows:

$$
\mathbf{Z}_{t}=\boldsymbol{\Lambda} \mathbf{X}_{t}+\left(\mathbf{I}_{m \times m}-\boldsymbol{\Lambda}\right) \mathbf{Z}_{t-1} \quad t=1,2, \ldots, n,
$$

where $\mathbf{Z}_{0}=\mathbf{0}, \boldsymbol{\Lambda}=\operatorname{diag}\left(\lambda_{1}, \lambda_{2}, \ldots, \lambda_{m}\right)$ and $\lambda_{j} \in(0,1]$ is a weighting parameter for the $j$-th component of $\mathbf{X}$, for $j=1,2, \ldots, m$, and $\mathbf{I}_{m \times m}$ is the identity matrix. The MEWMA chart is generally good for detecting relatively small shifts when $\lambda$ is chosen small, and it is good for detecting relatively large shifts when $\lambda$ is chosen large [19]. Literature recommends the use of the value of $\lambda$ between 0.2 and 0.3 for most monitoring purposes [32]. If there is no practical reason to give different weight for each component, then we can choose $\lambda_{1}=\lambda_{2}=\cdots=\lambda_{m}=\lambda$. In such cases,

$$
\mathbf{Z}_{t}=\lambda \mathbf{X}_{t}+(1-\lambda) \mathbf{Z}_{t-1}
$$

The MEWMA statistic, $\mathbf{V}_{t}^{2}$, can be computed recursively as follows [31]:

$$
\mathbf{V}_{t}^{2}=\mathbf{Z}_{i}^{T} \boldsymbol{\Sigma}_{\mathbf{Z}_{t}^{-1}}^{-} \mathbf{Z}_{t}
$$

where $\boldsymbol{\Sigma}_{\mathbf{Z}_{t}}$ is the covariance matrix of $\mathbf{Z}_{t}$. When $\lambda_{1}=\lambda_{2}=$ $\cdots=\lambda_{p}=\lambda$, the covariance matrix of $\mathbf{Z}_{t}$ can be rewritten as:

$$
\boldsymbol{\Sigma}_{\mathbf{Z}_{t}}=\frac{\lambda}{(2-\lambda)}\left[1-(1-\lambda)^{2 n}\right] \boldsymbol{\Sigma},
$$

where $\Sigma$ is the covariance matrix of the input data. The chart signals an anomaly when $\mathbf{V}_{t}^{2}>h$, where $h$ is the decision threshold. The value of $h$ can be computed via simulation to obtain specific threshold [33].

\section{EXPERIMENTS AND RESULTS}

In this section, we evaluate the abilities of the MEWMA monitoring chart to detect a fall.

\section{A. Data description}

To assess the detection performance of the proposed methods, we performed experiments on two publicly available fall detection databases: the UR fall detection dataset (URFD) and the fall detection dataset (FDD) [34], [35]. URFD comprises 70 sequences of several actions performed in different ways. Fall events and activities of daily living (ADL) events used in this work are recorded with an RGB camera. The URFD comprises 30 images per sequence for both classes: falls and typical ADLs. The second database is extracted from the FDD that comprises 191 video sequences with frame rate is 25 frames/s and a resolution of $320 \times 240$ pixels.

Anomaly detection performance is evaluated using the common criteria of missed detection rate (MDR) and false alarm rate (FAR). The MDR represents the number of anomalies that do not exceed the control limits (missed detection) over the total number of anomalies. The FAR is the number of false alarms over the total number of faultless data. The smaller the values of MDR and FAR, the better the performance of the corresponding monitoring chart [21].

\section{B. Detection results}

To test the performance of the fall detection scheme, two data sets, the URFD [34] and the FDD [35] has been used. First, the human body segmented using a background subtraction technique and area ratios are extracted from the posture in the image. Two cases of falls are performed. In the first case study, the testing data contains a true real fall or atypical event (case A). The testing data in the second case contains false falls, i.e., fall-like activities such as lying down (Case B). In all monitoring charts, the red-shaded area represents the region where the fall incident occurred in the testing data, and the dashed line while $95 \%$ control limits are plotted by the horizontal dashed line.

1) Case A - true fall:: Three examples are given here to show the capacity of the MEWMA monitoring chart to detect a true fall event. In the first example, the testing data used to test the performance of the MEWMA monitoring chart, which comprises 714 frames of a video sequence, contains a fall between frames 290 and 540. For the construction of the MEWMA chart, we choose $\lambda=0.2$ and a UCL of 10.97 so that its false alarm rate $\alpha=0.5 \%$. The results of the MEWMA chart are illustrated in Figure 5, and clearly show that this chart detect this fall event but it resulted in some false alarms (i.e., $\mathrm{FAR}=7.54 \%$ ) and missed detections (MDR=2\%).

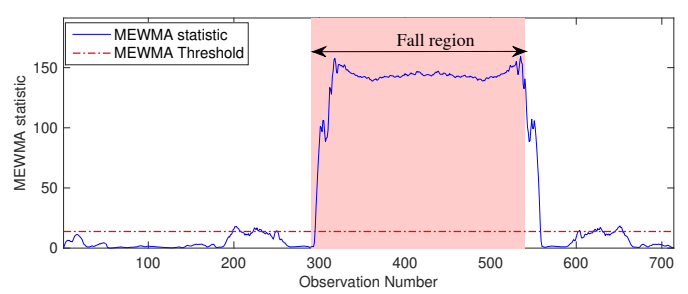

Fig. 5. The MEWMA chart when it is applied to the data considered in the first example of Case A and when $\lambda=0.2$.

In the second example, the testing data contains an anomaly or a fall event between frame numbers 430 and 495 . Monitoring results of MEWMA chart are shown in Figure 6. It successfully detects this fall but with some false alarms (i.e., $\mathrm{FAR}=11.16 \%$ ).

The aim of this third example was to assess the potential of the MEWMA monitoring chart to detect double falls. Towards 


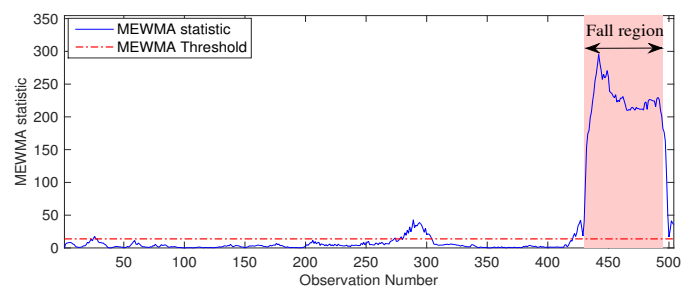

Fig. 6. The MEWMA chart when it is applied to the data considered in the second example of Case A and when $\lambda=0.2$.

this end, it is assumed that the testing data contains fall between frame interval [300, 510] and [570, 644]. Results of MEWMA anomaly detection algorithms for the considered testing data are shown in Figure 7. It can be seen that the MEWMA chart can detect these double falls but with several false alarms. This case study further testifies to the capability of the MEWMA for detecting falls.

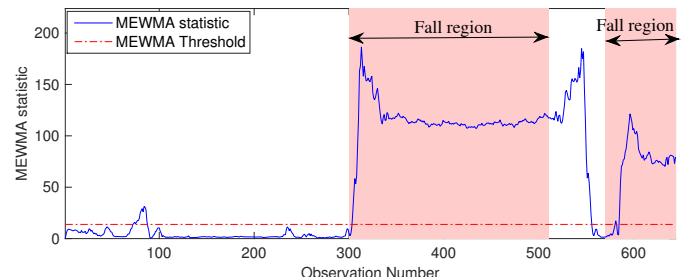

Fig. 7. The MEWMA chart when it is applied to the data considered in the third example of Case A and when $\lambda=0.2$.

2) Case B: False fall - Lying down: In this second case study, the testing data contains lying down or false fall between frame numbers 180 and 500. The results using the MEWMA chart illustrated in Figure 8, demonstrate that it could successfully detect this false fall but with several false alarms.

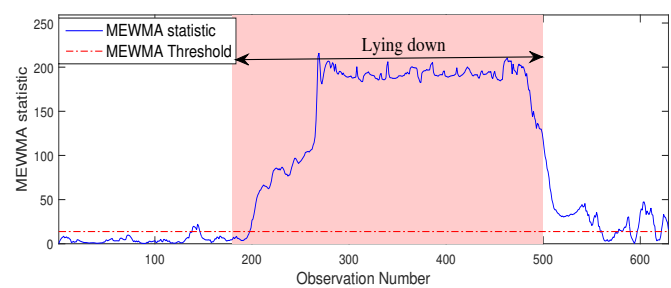

Fig. 8. The MEWMA chart when it is applied to the data considered in Case $\mathrm{B}$ and when $\lambda=0.2$.

Note that the MEWMA chart cannot differentiate real falls from certain fall-like activities such as lying down. To deal with this problem a classification module should be added after fall detection.

\section{CONCLUSION}

This paper addressed the detection of a human fall as a statistical anomaly detection problem. Specifically, the MEWMA monitoring chart was used towards this end based on variation in human silhouette shape. Two publicly available fall detection databases: the URFD and the FDD have been used to assess the ability of the MEWMA chart to detect a human fall. The results confirm the relevance and efficiency of the proposed solution for fall detection. The key advantage of this strategy is that it can be easily implemented in real time due to its low computational cost.

Our future work is focused on fall detection and classification (e.g., using support vector machine (SVM) to build a fall detection system that can accurately and quickly detect fall events. We plan to use SVM classifier to distinguish between true and false falls.

\section{ACKNOWLEDGEMENT}

This publication is based upon work supported by the King Abdullah University of Science and Technology (KAUST) Office of Sponsored Research (OSR) under Award No: OSR-2015-CRG4-2582. old people.

\section{REFERENCES}

[1] W. H. O. Ageing and L. C. Unit, WHO global report on falls prevention in older age. World Health Organization, 2008.

[2] R. Igual, C. Medrano, and I. Plaza, "Challenges, issues and trends in fall detection systems," Biomed. Eng. Online, vol. 12, no. 66, pp. 1-66, 2013.

[3] Y. Delahoz and M. Labrador, "Survey on fall detection and fall prevention using wearable and external sensors," Sensors, vol. 14, no. 10, pp. 19806-19842, 2014.

[4] M. Mubashir, L. Shao, and L. Seed, "A survey on fall detection: Principles and approaches," Neurocomputing, vol. 100, pp. 144-152, 2013.

[5] C. Rougier, J. Meunier, A. St-Arnaud, and J. Rousseau, "Robust video surveillance for fall detection based on human shape deformation," IEEE Transactions on Circuits and Systems for Video Technology, vol. 21, no. 5, pp. 611-622, 2011.

[6] L. Hazelhoff, J. Han et al., "Video-based fall detection in the home using principal component analysis," in Advanced Concepts for Intelligent Vision Systems. Springer, 2008, pp. 298-309.

[7] V. Vishwakarma, C. Mandal, and S. Sural, "Automatic detection of human fall in video," in Pattern Recognition and Machine Intelligence. Springer, 2007, pp. 616-623.

[8] C.-L. Liu, C.-H. Lee, and P.-M. Lin, "A fall detection system using k-nearest neighbor classifier," Expert systems with applications, vol. 37 , no. 10 , pp. 7174-7181, 2010.

[9] H. Rimminen, J. Lindström, M. Linnavuo, and R. Sepponen, "Detection of falls among the elderly by a floor sensor using the electric near field." IEEE transactions on information technology in biomedicine: a publication of the IEEE Engineering in Medicine and Biology Society, vol. 14 , no. 6, pp. 1475-1476, 2010.

[10] L. Y, H. K, and P. M, "A microphone array system for automatic fall detection," IEEE Transactions on Biomedical Engineering, vol. 59, no. 5, pp. 1291-1301, 2012.

[11] M. Aslan, A. Sengur, Y. Xiao, H. Wang, M. Ince, and X. Ma "Shape feature encoding via fisher vector for efficient fall detection in depth-videos," Applied Soft Computing, vol. 37, pp. 1023-1028, 2015.

[12] R. Gibson, A. Amira, N. Ramzan, P. C. de-la Higuera, and Z. Pervez, "Multiple comparator classifier framework for accelerometer-based fall detection and diagnostic," Applied Soft Computing, vol. 39, pp. 94-103, 2016.

[13] B. Kwolek and M. Kepski, "Fuzzy inference-based fall detection using kinect and body-worn accelerometer," Applied Soft Computing, vol. 40, pp. 305-318, 2016.

[14] C. Rougier, J. Meunier, A. St-Arnaud, and J. Rousseau, "Fall detection from human shape and motion history using video surveillance," in Advanced Information Networking and Applications Workshops, 2007, AINAW'07. 21st International Conference on, vol. 2. IEEE, 2007, pp. $875-880$. 
[15] E. Auvinet, F. Multon, A. Saint-Arnaud, J. Rousseau, and J. Meunier, "Fall detection with multiple cameras: An occlusion-resistant method based on 3-d silhouette vertical distribution," IEEE Transactions on Information Technology in Biomedicine, vol. 15, no. 2, pp. 290-300, 2011.

[16] L. Alhimale, H. Zedan, and A. Al-Bayatti, "The implementation of an intelligent and video-based fall detection system using a neural network," Applied Soft Computing, vol. 18, pp. 59-69, 2014.

[17] N. Zerrouki and A. Houacine, "Automatic classification of human body postures based on curvelet transform," in Image Analysis and Recognition. Springer, 2014, pp. 329-337.

[18] B. Kwolek and M. Kepski, "Improving fall detection by the use of depth sensor and accelerometer,' Neurocomputing, vol. 168, pp. 637-645, 2015.

[19] D. Montgomery, "Introduction to statistical quality control," John Wiley\& Sons, New York, 2005.

[20] F. Harrou, M. Nounou, H. Nounou, and M. Madakyaru, "PLS-based EWMA fault detection strategy for process monitoring," Journal of Loss Prevention in the Process Industries, vol. 36, pp. 108-119, 2015.

[21] F. Kadri, F. Harrou, S. Chaabane, Y. Sun, and C. Tahon, "Seasonal ARMA-based SPC charts for anomaly detection: Application to emergency department systems," Neurocomputing, vol. 173, pp. 2102-2114, 2016.

[22] F. Harrou, Y. Sun, and S. Khadraoui, "Amalgamation of anomaly-detection indices for enhanced process monitoring," Journal of Loss Prevention in the Process Industries, vol. 40, pp. 365-377, 2016.

[23] S. Qin, "Statistical process monitoring: Basics and beyond," Journal of Chemometrics, vol. 17, no. 8/9, pp. 480-502, 2003.

[24] a. L. W. H. Abdi, "Principal component analysis," Wiley Interdisciplinary Reviews: Computational Statistics, vol. 2, no. 4, pp. 433-459, 2010.

[25] J. MacGregor and T. Kourti, "Statistical process control of multivariate processes," Control Engineering Practice, vol. 3, no. 3, 1995.

[26] A. Elgammal, D. Harwood, and L. Davis, "Non-parametric model for background subtraction," in Computer Vision-ECCV 2000. Springer 2000, pp. 751-767.

[27] K. Kim, T. Chalidabhongse, D. Harwood, and L. Davis, "Real-time foreground-background segmentation using codebook model," Real-time imaging, vol. 11, no. 3, pp. 172-185, 2005.

[28] I. Guyon and A. Elisseeff, "An introduction to feature extraction," in Feature extraction. Springer, 2006, pp. 1-25.

[29] H. Liu and C. Zuo, "An improved algorithm of automatic fall detection," AASRI Procedia, vol. 1, pp. 353-358, 2012.

[30] F. Harrou and M. Nounou, "Monitoring linear antenna arrays using an exponentially weighted moving average-based fault detection scheme," Systems Science \& Control Engineering: An Open Access Journal, vol. 2, no. 1, pp. 433-443, 2014

[31] C. A. Lowry, W. H. Woodall, C. W. Champ, and S. E. Rigdon, "A multivariate exponentially weighted moving average control chart," Technometrics, vol. 34, no. 1, pp. 46-53, 1992.

[32] J. S. Hunter, "The exponentially weighted moving average." Journal of Quality Technology, vol. 18, no. 4, pp. 203-210, 1986.

[33] K. M. Bodden and S. E. Rigdon, "A program for approximating the in-control ARL for the MEWMA chart," Journal of Quality Technology, vol. 31, no. 1, pp. 120-123, 1999 .

[34] B. Kwolek and M. Kepski, "Human fall detection on embedded platform using depth maps and wireless accelerometer," Computer methods and programs in biomedicine, vol. 117, no. 3, pp. 489-501, 2014.

[35] I. Charfi, J. Miteran, J. Dubois, M. Atri, and R. Tourki, "Definition and performance evaluation of a robust SVM based fall detection solution,' in Signal Image Technology and Internet Based Systems (SITIS), 2012 Eighth International Conference on. IEEE, 2012, pp. 218-224. 